\title{
Retraction: clinical and technical phosphoproteomic research
}

\author{
Elena López ${ }^{1 *}$, Isabel López ${ }^{2}$, Antonio Ferreira ${ }^{3}$ and Julia Sequí ${ }^{4}$
}

\section{Retraction notice}

This article [1] has been regretfully retracted by the Editors because of significant overlap with a figure and text from previously published articles [2-4]. We apologise to all affected parties for the inconvenience caused.

\footnotetext{
Author details

${ }^{1}$ Inflammatory core, Centro de Investigación i+12 del Hospital Universitario 12 de Octubre, Avda de Córdoba s/n, 28041, Madrid, Spain. ${ }^{2}$ Hematology Department, Hospital Universitario 12 Octubre, Avda de Córdoba s/n, 28041, Madrid, Spain. ${ }^{3}$ Immunology Department, Hospital Universitario La Paz, P de la Castellana, 261-28046, Madrid, Spain. ${ }^{4}$ Immunology Department, Hospital

Carlos III, Sinesio Delgado, 28029, Madrid, Spain.
}

Received: 28 March 2013 Accepted: 28 March 2013

Published: 23 April 2013

\section{References}

1. Lopez E, Lopez I, Ferreira A, Sequi J: Clinical and technical phosphoproteomic research. Proteome Sci 2011, 9(1):27.

2. López E, Matthiesen R, López I, Ashman K, Mendieta J, Wesselink JJ, GómezPuertas P, Ferreira A: Functional phosphoproteomics tools for current immunological disorders research. Journal of integrated omics 2011, 1(1):1-16.

3. Ashman K, Villar EL: Phosphoproteomics and cancer research. Clin Trans/ Oncol 2009, 11(6):356-62.

4. Choudhary C, Mann M: Decoding signalling networks by mass spectrometry-based proteomics. Nat Rev Mol Cell Biol 2010, 11:427-439.

\footnotetext{
* Correspondence: elena.lopez.villar@gmail.com

${ }^{1}$ Inflammatory core, Centro de Investigación i+12 del Hospital Universitario

12 de Octubre, Avda de Córdoba s/n, 28041, Madrid, Spain

Full list of author information is available at the end of the article
}

\section{Submit your next manuscript to BioMed Central and take full advantage of:}

- Convenient online submission

- Thorough peer review

- No space constraints or color figure charges

- Immediate publication on acceptance

- Inclusion in PubMed, CAS, Scopus and Google Scholar

- Research which is freely available for redistribution

Submit your manuscript at www.biomedcentral.com/submit 